\title{
AÇÃO DOS ANTIOXIDANTES NO TRATAMENTO DE PACIENTES ONCOLÓGICOS SUBMETIDOS A QUIMIOTERÁPIA E/OU RADIOTERÁPIA - UMA REVISÃO INTEGRATIVA
}

\section{ACTION OF THE ANTIOXIDANTS IN TREATMENT OF ONCOLOGIC PATIENTS SUBMITTED TO CHEMOTHERAPY AND/OR RADIOTHERAPY - AN INTEGRATIVE REVIEW}

\author{
Maxwelly Alves dos Santos \\ Nutricionista, Faculdade Presidente Antônio Carlos \\ UNIPAC de Teófilo Otoni, Brasil. \\ E-mails: maxwellyalvesdossantos@gmail.com
}

Natany Gomes Dias

Nutricionista, Faculdade Presidente Antônio Carlos

UNIPAC de Teófilo Otoni, Brasil.

E-mails: natany.g.dias7@gmail.com

\section{Karine Rodrigues da Silva Neumann}

Nutricionista especialista em Vigilância em Saúde, Faculdade Presidente Antônio Carlos UNIPAC de Teófilo Otoni, Brasil.

E-mails: krsnut@yahoo.com.br

Paloma Benigno de Morais

Bióloga mestre em Ciências biológicas, Faculdade Presidente Antônio Carlos UNIPAC de Teófilo Otoni, Brasil.

E-mails: palomabenigno@gmail.com

Recebido: 00/00/0000 - Aceito: 00/00/0000

\section{Resumo}

O presente estudo tem como objetivo identificar a ação de antioxidantes no tratamento de pacientes oncológicos submetidos à quimioterapia e/ou radioterapia. O tratamento do câncer inclui radioterapia e quimioterapia, que por serem agressivos evolui com efeitos colaterais que podem causar sérios danos ao organismo. Estudos mostram que os antioxidantes podem amenizar esses danos. Antioxidantes são compostos que podem retardar ou inibir a oxidação de lipídios ou têm como função combater radicais livres evitar processos inflamatórios, melhorar o sistema imune e atuar na reconstrução celular de células danificadas, como acontece com pacientes com câncer em tratamento quimioterápico e/ou 
radioterápico. Foram incluídos neste estudo pesquisas apresentadas em foram de resumo ou artigo no período de 2010 a 2019, nos idiomas português e inglês, disponível nas bases de dados gratuitas e dispostos na forma íntegra para o acesso, totalizando 6 trabalhos. Os resultados mostram que os antioxidantes mais estudados no tratamento de pacientes oncológicos em rádio e/ou quimioterapia são as Vitaminas $A, C, D, E$, além da Curcumina e Ubiquinona e que apesar de haver controvérsias entre alguns estudos, a maioria demonstra resultado positivo na evolução do paciente, reduzindo a mortalidade, melhorando quadros de dermatite causada pela radioterapia. Sendo assim é importante que sejam realizados mais estudos experimentais e com mais tipos de antioxidantes tendo em vista a importância dos mesmos na evolução dos pacientes oncológicos. A metodologia aplicada foi uma revisão integrativa da literatura, através de resumos e artigos experimentais sobre o tema, disponíveis nas bases de dados eletrônicos: PUBMED, Scientific Electronic Library OnLine (SCIELO) e Instituto Nacional do Câncer (INCA).

Palavras-chave: Câncer; Radioterapia; Quimioterapia; Antioxidantes.

\section{Abstract}

The aim of present study was to identify the action of antioxidants in the treatment of cancer patients undergoing chemotherapy and / or radiotherapy. Cancer treatment includes radiotherapy and chemotherapy, which are aggressive and have side effects that can cause serious damage to the body. Studies show that antioxidants can alleviate this damage. Antioxidants are compounds that can slow or inhibit lipid oxidation or have the function of fighting free radicals, preventing inflammatory processes, improving the immune system and acting on cell reconstruction of damaged cells, as happens with cancer patients undergoing chemotherapy and / or radiotherapy. We included in this study research presented in were abstract or article from 2010 to 2019, in Portuguese and English, available in free databases and arranged in full for access, totaling 6 papers. The results show that the most studied antioxidants in the treatment of cancer patients in radiotherapy and / or chemotherapy are Vitamins $A, C, D, E$, besides Curcumin and Ubiquinone, although there is controversy among some studies, most of them show positive results. patient evolution, reducing mortality, improving dermatitis caused by radiotherapy. Therefore, it's important that more experimental studies with more types of antioxidants be carried out in view of their importance in the evolution of cancer patients. The methodology applied was an integrative literature review, through abstracts and experimental articles on the subject, available in electronic databases: PUBMED, Scientific Electronic Library OnLine (SCIELO) and National Cancer Institute (INCA).

Keywords: Cancer; Radiotherapy; Chemotherapy; Antioxidants

\section{Introdução}

O câncer tem sido uma das principais causas de morte devido a doenças não transmissíveis em todo o mundo e, portanto, é um grande problema de saúde 
desenvolvimento. Estima-se, no biênio 2018-2029, a ocorrência de 600 mil casos novos de câncer segundo o Instituto nacional do Câncer (INCA).

Os meios de tratamentos mais utilizados nessa doença é a cirurgia para a retirada do tumor, radioterapia e quimioterapia, mas por serem tratamentos muito agressivos e invasivos seus efeitos colaterais podem causar sérios danos ao organismo.

A quimio e radioterapia são tratamentos essenciais, mas que produzem efeitos sintomáticos debilitando ainda mais o paciente oncológico. Os efeitos colaterais da quimioterapia podem ser de forma aguda, ou seja, logo após a administração da droga e podendo durar dias e a forma tardia, que ocorre dias ou meses após a ingestão da droga. Já o tratamento radioterapêutico, importante no controle local do câncer, permite melhoria nos resultados e diminuição dos efeitos adversos em tecidos sadios, mas essa técnica não aumenta a sobrevida do paciente. (REFEPADILHA, 2004)

Quanto mais avançada a doença menor é a concentração de antioxidantes no organismo que está relacionado à redução de enzimas que são afetadas pelo tratamento radioterápico. Estudos mostram que os antioxidantes podem amenizar esses efeitos. (SAMPAIO, 2009)

Antioxidantes, compostos que podem retardar ou inibir a oxidação de lipídios, têm como função combater radicais livres, evitar processos inflamatórios, melhorar o sistema imune e atuar na reconstrução celular de células danificadas, como acontece com pacientes com câncer em tratamento quimioterápico e/ou radioterápico. (SANTOS; SILVA, 2016)

Mesmo presentes em baixas concentrações, os nutrientes antioxidantes são substâncias que possuem a capacidade de retardar ou impedir as taxas de oxidação, a aplicação desses nutrientes pra tratamento oncológico tem sido objeto de muitas pesquisas, onde os resultados têm mostrado êxito quando utilizados juntamente com medicação e terapia adequada. (SAMPAIO, 2009)

Dentro desse contexto, o objetivo do presente estudo é através de uma revisão integrativa identificar a ação de antioxidantes no tratamento de pacientes oncológicos submetidos à quimioterapia e/ou radioterapia.

\section{Passos metodológicos}

O presente estudo trata-se de uma revisão integrativa por nos possibilitar reunir e sintetizar informações comprovadas cientificamente e que são de extrema importância sobre o tema proposto, por meio de um levantamento bibliográfico com a 
utilização de estudos não experimentais e estudos experimentais para atender ao tema investigado, sendo esta a mais ampla explanação metodológica referente às revisões.

Foi realizada uma revisão integrativa em seis etapas: seleção da questão norteadora; definição das características das pesquisas primárias; seleção das pesquisas que compuseram a amostra; análise dos artigos; interpretação dos resultados; e o exame crítico dos achados, com a seguinte questão norteadora: "Quais os efeitos do uso dos nutrientes antioxidantes para pacientes oncológicos submetidos ao tratamento quimio e/ou radioterápico?"

A coleta de dados ocorreu durante os meses de agosto a novembro de 2019. Para consolidação do estudo foi realizada seleção de referenciais nas bases de dados eletrônicos: National Library of Medicine (MEDLINE), Instituto Nacional do Câncer (INCA), Scientific Electronic Library Online (SCIELO), PUBMED, LILACS e demais bases visando atender a recomendação da literatura de que se busquem diferentes fontes para o levantamento de publicações.

Foram utilizados os termos "Antioxidantes", "Câncer" e "Quimioterapia", "Radioterapia" e "Efeitos Colaterais" e "Estresse Oxidativo".

Foram incluídas neste estudo pesquisas apresentadas em forma de artigo no período de 2009 a novembro de 2018, no idioma português e inglês disponível nas bases de dados gratuitas e dispostos na forma íntegra para o acesso.

É importante ressaltar que os estudos encontrados em mais de uma das bases de dados utilizadas foram considerados somente uma vez, sendo contabilizados na base que apresentou maior número de trabalhos.

A análise dos dados foi realizada de forma descritiva, procedendo-se à categorização dos dados extraídos dos estudos selecionados em grupos temáticos, a partir da identificação de variáveis de interesse e conceitos-chave, conforme proposto em literatura específica acerca de revisão integrativa de literatura.

\section{Alguns antioxidantes}

Antioxidantes são compostos que podem retardar ou inibir a oxidação de lipídios ou outras moléculas, evitando o início ou propagação das reações em cadeia de oxidação. A atividade antioxidante de compostos fenólicos é principalmente devida às suas propriedades de óxidoredução, as quais podem desempenhar um importante papel na absorção e neutralização de radicais livres, quelando o oxigênio triplete e 
defesa contra as espécies reativas de oxigênio, que são responsáveis por danos ao DNA, regulação da diferenciação celular e, consequentemente, inibição do crescimento de células cancerígenas. (REFEPADILHA, 2004)

Os nutrientes antioxidantes aumentam 0 efeito dos antineoplásicos, ocasionando redução do tumor, em consequência, na mínima produção de efeitos inversos, proporcionando melhoria na qualidade e prolongamento da vida, mesmo em uma quantidade pequena, retarda ou impede a oxidação; e são definidos como enzimáticos, que são produzidos pelo próprio organismo; e os não enzimáticos, as vitaminas A, C e E e minerais. (SANTOS; SILVA, 2016)

Os antioxidantes são compostos que possuem a capacidade de inibir ou atrasar as taxas de oxidação de lipídios, ou outras moléculas, prevenindo a gênese ou proliferação das reações em cadeia de oxidação. A ação antioxidante de compostos fenólicos é especialmente por causa das suas propriedades de óxido-redução, estas, que podem desenvolver um papel importante neutralizando e absorvendo radicais livres, quelar o oxigênio singlete e triplete ou dissociando peróxidos. Entre as ações das vitaminas e minerais, uma é a de defesa contra espécies reativas de oxigênio, que por sua vez, são responsáveis por causar danos no DNA, regular a diferenciação celular e como consequência, inibir o crescimento de células cancerígenas. (REFEPADILHA \& SAMPAIO, 2009)

Entre os micronutrientes mais investigados por sua atuação quimiopreventiva na carcinogênese é importante ressaltar as vitaminas antioxidantes, sendo as vitaminas $\mathrm{A}$ C e E.

A vitamina A foi a primeira vitamina lipossolúvel a ser reconhecida em 1913 e o pcaroteno é o carotenóide encontrado na natureza com maior poder de formação de vitamina $A$, sendo capaz de conferir proteção contra diversos tipos de tumores. Entre as suas funções está a capacidade de inibir a oxidação de compostos pelos peróxidos. O mecanismo pelo qual estas substâncias protegem os sistemas biológicos contra os danos mediados pelos Radicais Livres $(R L)$ parece depender da taxa de repressão da reação de formação dos RL. (SANTOS; CRUZ, 2001)

O ácido ascórbico é uma vitamina hidrossolúvel e antioxidante que reage diretamente com o oxigênio simples, radical hidroxila e radical superóxido, além de regenerar a vitamina $\mathrm{E}$. Além disto, esta vitamina mantém as enzimas tiols em seus estados reduzidos e poupa a glutationaperoxidase, que é um importante antioxidante intracelular e cofator enzimático. (JUNIOR, 2001)

A vitamina $E$ é outro antioxidante dietético de grande importância, e sua 
das células malignas de linfomas de câncer. Sua função como antioxidante é proteger os tecidos adiposos do ataque de radicais livres,como por exemplo, a formação de radicais peróxidos a partir de ácidos graxos poliinsaturados nas membranas fosfolipídicas. Em estados de deficiência desta vitamina, os danos celulares causados pela produção de radicais livres pelo tumor causam a destruição celular. (ROHENKOHL, 2011)

O cúrcuma, conhecido como açafrão-da-terra ou apenas açafrão, tem como substância ativa, a curcumina. A substância tem sido muito estudada como potente adjuvante ao tratamento oncológico, com o objetivo de diminuir os efeitos colaterais das terapias e como potente atividade anti-inflamatória, quimioprotetora, imunomodulatória, antioxidante e antiproliferativa, apoptótico, antiangiogênico, antimetastático. (FIGUEREDO, 2018)

\section{Resultados e discussão}

No geral foram encontrados 854 artigos nas diversas bases, que após análise foram selecionados 6 artigos, sendo 3 no PUBMED, 2 no INCA e 1 SCIELO.

Para a realização deste estudo foram utilizados estudos experimentais e não experimentais, utilizamos artigos que relatassem a ação dos antioxidantes sobre efeitos adversos do tratamento de quimioterapia e/ ou radioterapia. Os artigos utilizados podem se diferenciar um do outro pelos tipos e os efeitos dos antioxidantes utilizados, o tipo de tratamento e o tipo de câncer apresentado pelo paciente.

Realizado no período de março de 2002 a abril de 2006, um estudo de coorte utilizando mulheres com idade média entre 20 a 70 anos diagnosticadas com câncer de mama na cidade de Xangai, China buscou avaliar os suplementos vitamínicos durante o tratamento e sobrevida dos pacientes. A utilização dos antioxidantes foi feita nos primeiros seis meses após o diagnóstico. A ação dos antioxidantes reduziu em $18 \%$ a mortalidade em mulheres que fizeram uso dos antioxidantes (vitaminas C, E e multivitamínicos) e redução de $22 \%$ no risco de recorrência. (NECHUTA et al, 2010)

Um estudo randomizado com duração de cinco anos procurou analisar a ação dos antioxidantes vitaminas $\mathrm{C}$ e $\mathrm{E}$ sobre os efeitos nocivos das drogas anticâncer utilizados por pacientes com câncer de mama. Ao contrário do grupo que utilizou a quimioterapia isoladamente, o grupo que foi suplementado pela vitamina $C$ e $E$ apresentou valores normais nos níveis de biomarcadores. As atividades enzimáticas 
aumentaram significativamente. A ação da suplementação das vitaminas C e E restaurou a condição de antioxidantes no organismo e reduziu os danos ao DNA que foram diminuídos pela quimioterapia.

Com o objetivo de analisar a capacidade da curcumina em diminuir a gravidade da dermatite causada pela radioterapia em 30 mulheres com câncer de mama com idade média de 58 anos, foi realizado um estudo randomizado clínico duplo cego controlado por placebo. Foram administradas a pacientes 2,0 gramas de cúrcuma ou placebo três vezes (cerca de 6,0 gramas ao dia) de forma oral durante todo o período de radioterapia. A cada semana as pacientes passavam por uma avaliação onde pontuava a gravidade da dermatite de radiação, conferição da vermelhidão onde os sinais eram retratados em imagens digitais e questionários de auto-relato. $A$ ação da curcumina administrada por via oral apresentou melhora da dermatite causada pela radiação. (RYAN et al., 2013)

Um estudo desenvolvido com o objetivo de revisar estudos experimentais clínicos feitos em pacientes com câncer, envolveu o consumo de antioxidantes durante o período de tratamento quimio e radioterápico, no período de 1982 a 2014. Foram inclusos artigos feitos com pessoas que apresentavam qualquer tipo de câncer, submetidos aos tratamentos quimio e/ou radioterápico e utilizando suplementos antioxidantes orais: ubiquinona, carotenóides, vitaminas A, E, C, B9 e selênio. Após análise constatou-se que os pacientes com uso de antioxidantes apresentaram redução nos níveis de proteínas carboniladas que são marcadores padrão do estresse oxidativo e melhoraram a capacidade cognitiva global. As Vitaminas C e E também diminuíram os danos no DNA causados pela quimioterapia. (YASUEDA; URUSHIMAET, 2015)

Com o objetivo de conhecer, analisar e definir a ação da curcumina em pacientes oncológicos foi realizado um estudo baseado em uma revisão de literatura. O material utilizado para a construção da pesquisa foi recolhido em bancos de dados eletrônicos como PUBMEB, INCA e outros. Nos estudos retratados utilizaram-se células cancerígenas mantidas em processos bioquímicos, constatando cientificamente que a curcumina possui propriedades inibidoras na multiplicação, crescimento e expansão de tumores e autodestruição de celular. Constatou-se também que a Curcumina numa quantidade de 5 a 6 gramas diariamente possui efeito antioxidante, anticancinogênico, imunomodelador, anti-inflamatório e quimioprotetor. Sua suplementação pode ser utilizada com segurança por pacientes oncológicos. (FIGUEREDO; SAMPAIO, 2009) 
Um outro estudo foi realizado com a finalidade de avaliar a utilização da Vitamina $\mathrm{E}$ na quimioproteção contra os danos toxicogenéticos de antineoplásicos onde se utilizou a concentração de $100 \mathrm{UI}$ da vitamina E sobre as atividades danosas, toxidades e mutações de células causadas por substâncias quimioterápicas. A suplementação com a vitamina $E$ diminuiu danos oxidativos no citoplasma e na mitocôndria causados pelos Antineoplásicos que são medicamentos utilizados na quimioterapia para destruir células malignas. Os danos causados pelos quimioterápicos no gene apresentaram valores significadamente diminuídos quando associaram a vitamina $E$ ao tratamento. $O$ estudo apresentou significância na ação da vitamina $E$ sobre as toxidades celulares e mutações genéticas, protegendo $O$ organismo da variabilidade e mutabilidade genética. (FERNANDES, 2018)

A partir da análise dos textos selecionados, os dados foram organizados em um quadro (Quadro 1), que mostra a distribuição dos estudos incluídos na revisão integrativa, de acordo com autores e ano de publicação, título, base de dados, antioxidante utilizado e ação:

Quadro 1 - Distribuição dos estudos incluídos na revisão integrativa, de acordo com autores e ano de publicação, título, base de dados, antioxidantes e ação.

\begin{tabular}{|c|c|c|c|c|}
\hline $\begin{array}{l}\text { AUTORES E } \\
\text { ANO DE } \\
\text { PUBLICAÇÃO }\end{array}$ & TÍTULO & $\begin{array}{l}\text { BASE } \\
\text { DE } \\
\text { DADOS }\end{array}$ & ANTIOXIDANTE & AÇÃO \\
\hline $\begin{array}{l}\text { Nechuta S , Lu } \\
\text { W et al } \\
(2010)\end{array}$ & $\begin{array}{l}\text { Vitamin } \\
\text { supplement use } \\
\text { during breast } \\
\text { cancer } \\
\text { treatment and } \\
\text { survival: a } \\
\text { prospective } \\
\text { cohort study }\end{array}$ & PUBMED & $\begin{array}{l}\text { Vitamina E, C e } \\
\text { Multivitaminas }\end{array}$ & $\begin{array}{lr}\text { Redução } & \text { dos } \\
\text { níveis } & \text { de } \\
\text { mortalidade } & \text { em } \\
18 \% & \text { nas } \\
\text { mulheres } & \text { que } \\
\text { faziam } & \\
\text { quimioterapia e } \\
\text { em 22\% } & \text { do } \\
\text { risco } & \text { de } \\
\text { recorrência } & \end{array}$ \\
\hline
\end{tabular}




\begin{tabular}{|c|c|c|c|c|}
\hline $\begin{array}{l}\text { Suhail N, Bilal } \\
\text { N, Khan HY, } \\
\text { Hasan S, Sharma } \\
\text { S et al } \\
(2011)\end{array}$ & $\begin{array}{l}\text { Effect of } \\
\text { vitamins } C \text { and } \\
E \text { on antioxidant } \\
\text { status of breast- } \\
\text { cancer patients } \\
\text { undergoing } \\
\text { chemotherapy }\end{array}$ & PUBMED & Vitamina C e E & $\begin{array}{l}\text { Restauração } \\
\text { da condição de } \\
\text { antioxidantes } \\
\text { no organismo e } \\
\text { redução dos } \\
\text { danos ao DNA } \\
\text { que foram } \\
\text { diminuídos } \\
\text { pela } \\
\text { quimioterapia }\end{array}$ \\
\hline $\begin{array}{l}\text { Julie L. } \\
\text { Ryan ,Charles } \\
\text { EHeckler et al. } \\
(2013)\end{array}$ & $\begin{array}{l}\text { Curcumin for } \\
\text { Radiation } \\
\text { Dermatitis: A } \\
\text { randomized, } \\
\text { double-blind, } \\
\text { placebo- } \\
\text { controlled } \\
\text { clinical study of } \\
30 \text { breast cancer } \\
\text { patients }\end{array}$ & PUBMED & Curcumina & $\begin{array}{ll}\text { Melhora } & \text { da } \\
\text { dermatite } & \\
\text { causada pela } \\
\text { radiação }\end{array}$ \\
\hline $\begin{array}{l}\text { Asuka } \\
\text { Yasueda } \\
\text {,HayatoUrushimaet } \\
\text { et al. }\end{array}$ & $\begin{array}{l}\text { Eficácia e } \\
\text { interação de } \\
\text { suplementos } \\
\text { antioxidantes } \\
\text { como terapia }\end{array}$ & SCIELO & $\begin{array}{l}\text { Ubiquinona, } \\
\text { carotenóides, } \\
\text { vitaminas A, E, } \\
\text { C, B9 e selênio }\end{array}$ & $\begin{array}{l}\text { Redução dos } \\
\text { níveis de } \\
\text { proteínas } \\
\text { carboniladas e } \\
\text { dos danos ao } \\
\text { DNA }\end{array}$ \\
\hline
\end{tabular}

\begin{tabular}{|c|c|c|c|c|}
\hline (2015) & $\begin{array}{ll}\text { adjuvante } & \text { no } \\
\text { tratamento } & \text { do } \\
\text { câncer } & \\
\end{array}$ & & & \\
\hline $\begin{array}{ll}\text { Abdias } & \text { Vieira } \\
\text { de } & \text { Mélo } \\
\text { Figueredo. } \\
(2018)\end{array}$ & $\begin{array}{ll}\begin{array}{l}\text { Cúrcuma } \\
\text { (cúrcuma }\end{array} \\
\text { longa } & \text { L): } \\
\text { Benefícios } & \text { na } \\
\text { Prevenção } & \text { e }\end{array}$ & $\begin{array}{l}\text { Instituto } \\
\text { Nacional } \\
\text { do } \\
\text { Câncer } \\
\text { (INCA) }\end{array}$ & Curcumina & $\begin{array}{l}\text { Efeito } \\
\text { antioxidante, } \\
\text { anticancinogênico, } \\
\text { imunomodelador, } \\
\text { anti-inflamatório e }\end{array}$ \\
\hline
\end{tabular}




\begin{tabular}{|l|l|l|l|l|}
\hline & $\begin{array}{l}\text { Tratamento do } \\
\text { Câncer }\end{array}$ & & & quimioprotetor \\
& & & \\
\hline Andressa & Utilização da & Instituto & Vitamina E & Diminuição dos \\
Naiane & Vitamina E na & Nacional & & danos oxidativos \\
Rodrigues & Quimioproteção & do & & no citoplasma e \\
Monteiro & contra os & Câncer & & na mitocôndria \\
Fernandes, et & Danos pelos \\
al. & Toxicogenéticos & (INCA) & & causados Antineoplásicos \\
(2018) & de & & \\
& Antineoplásicos & & \\
\hline & em Estudos não & & \\
\hline
\end{tabular}

Por meio dos estudos é possível perceber que os antioxidantes mais estudados em pacientes em quimio e/ou radioterapia são Vitaminas $A, C, D, E$, além da Curcumina e Ubiquinona foi encontrada associação positiva do uso destas substancias no tratamento desses pacientes como o combate as toxicidades, mutações no gene e danos no DNA, redução dos níveis de proteína carboniladas que são marcadores padrão do estresse oxidativo e combate significativo sobre a dermatite que é uma manifestação frequente em pacientes que fazem radioterapia, sendo ocasionada dela radiação.

Revista Multidisciplinar do Nordeste Mineiro, v...., n..., p. 01-X, 2018/02 ISSN 2178-6925 / doi.org/10.17648/2178-6925

\section{Considerações finais}

Os pacientes oncológicos costumam apresentar muitos sintomas adversos a quimio e radioterapia devido aos agentes antineoplásicos e à radiação presentes nesses tratamentos. A intervenção nutricional é imprescindível principalmente nessa fase, uma vez que esses efeitos colaterais podem comprometer ainda mais o quadro clínico do paciente diminuindo suas chances de sobrevida. 
É de suma importância analisar a situação nutricional do paciente, ofertar uma alimentação saudável e minimizar os desconfortos causados pelo tratamento e promover uma melhor qualidade de vida ao paciente, pois o consumo alimentar abaixo do recomendado em pacientes com câncer pode causar maiores danos a saúde e comprometer ainda mais o estado nutricional e clínico do paciente.

Uma alimentação fonte de nutrientes antioxidantes sendo ingerida diariamente, além de, realizar suas funções básicas nutricionais pode combater radicais livres evitando diversos malefícios ao organismo, podendo esses ser consumidos por meio da alimentação ou por meio de suplementos administrados via oral.

Dentro desse contexto está o Nutricionista, o único profissional capacitado tanto para avaliar nutricionalmente o paciente com câncer em tratamento quimio ou radioterápico quanto prescrever uma alimentação específica para cada caso, incluindo antioxidantes que vão minimizar os danos causados pelo tratamento.

Mesmo com a eficácia dos antioxidantes comprovada, alguns estudos associam a utilização dos antioxidantes no período de tratamento com a diminuição da eficácia do tratamento com a justificativa que essas substâncias ao exercer sua função acabam protegendo também células tumorais, por seu efeito protetor celular ser exponencial .

Apesar da grande quantidade de estudos relacionando os antioxidantes com o câncer, é necessária a produção de mais estudos, principalmente ensaios clínicos randomizados que estejam direcionados a avaliar a ação desta substância nos efeitos adversos dos tratamentos, utilizando um tipo de antioxidante específico, para assim avaliar os benefícios e se eles irão apresentar alguma contra indicação.

\section{Referências}

FERNANDES, A.N.R.M. Utilização da vitamina E na quimioproteção contra os danos toxicogenéticos de antineoplásicos em estudos não clínicos. et al. 2018 
FIGUEREDO, A.V.M. Cúrcuma (cúrcuma longa L): Benefícios na Prevenção e Tratamento do Câncer. 2018 [acesso em 2019 nov 02].

Disponível em <http://www1.inca.gov.br/rbc/anais/64-2-suplemento-3.pdf>

JÚNIOR, L.R.; HOER, N.F.; VELLASCO, A.P. Sistema antioxidante envolvendo o ciclo metabólico da glutationa associado a métodos eletroanalíticos na avaliação do estresse oxidativo. Quím. Nova, UNICAMP, vol.24 no.1 São Paulo. 2001[acesso em 2019 out 20].

Disponível em <http://www.scielo.br/scielo.php?script=sci_arttext\&pid=S010040422001000100019>

NECHUTA S, L.W.; CHEN, Z.; ZHENG, Y. ;CAI, H. et al. Vitamin supplement use during breast cancer treatment and survival: a prospective cohort study.2010 [acesso em 2019 out 20]. Disponível em <https://www.ncbi.nlm.nih.gov/pubmed/21177425>

REFEPADILHA, P C., PINHEIRO, R. L. O Papel dos Alimentos Funcionais na Prevenção e Controle do Câncer de Mama Revista Brasileira de Cancerologia. Rio de janeiro RJ, 2004 [acesso em 2019 out 20]; 50(3): 251-260. Disponível em <http://www1.inca.gov.br/rbc/n_50/v03/pdf/REVISAO3.pdf>

ROHENKOHL, C.C.; CARNIEL, A.P.; COLPO, E. Consumo de antioxidantes durante tratamento quimioterápico.São Paulo $A B C D$, arq. bras. cir. dig. vol.24 no.2 São Paulo. 2011[acesso em 2019 out 05]. Disponivel em $<$ http://www.scielo.br/scielo.php?script=sci_arttext\&pid=S0102-

67202011000200004>

RYAN, J.L.; HECKLER, C.E.; LING, M.N. WILLIAMS, J.P.; PETLAND, A.P.; MOROW GR. Curcumin for Radiation Dermatitis: A randomized, double-blind, placebo-controlled clinical study of $\mathbf{3 0}$ breast cancer patients. 2013 [acesso $\begin{array}{lllll} & 2019 & \text { out } & 20] & \text { Disponível }\end{array}$ <https://www.ncbi.nlm.nih.gov/pmc/articles/PMC3998827/>

SAMPAIO, L.C.; ALMEIDA, C.F. Vitaminas antioxidantes na prevenção do câncer do colo uterino revista brasileira de cancerologia. 2009 [acesso em: 2019 out 25]; 55(3): 289-296. Disponível em<http://www1.inca.gov.br/rbc/n_55/v03/pdf/93_revisao_literatura4.pdf>

SANTOS, H.S.; CRUZ, W.M.S. Terapia nutricional com vitaminas antioxidantes e o tratamento quimioterápico oncológico, Revista Brasileira de Cancerologia, 2001 [acesso em 2019 out 05]; 47(3): 303-0

Disponível em <http://www.cookie.com.br/site/wp-content/uploads/2015/03/VitaminasAntioxidantes-e-o-Tratamento-Quimioter\%C3\%A1 pico-Oncol\%C3\%B3gico.pdf> 
SANTOS, S.E.M.; SILVA M.L.S. Estado nutricional e ingestão alimentar de pacientes com câncer durante o tratamento quimioterápico em um instituto de oncologia e radioterapia de Porto Velho. RO, Porto velho RO, Centro de ensino São Lucas 2016 [acesso em: 2019 out 26]. Disponível em: http://repositorio.saolucas.edu.br.

SUHAIL, N.; BILAL, N.; KHAN, H.Y, ET AL. Effect of vitamins $\mathbf{C}$ and $\mathbf{E}$ on antioxidant status of breast-cancer patients undergoing chemother Effect of vitamins $C$ and $E$ on antioxidant status of breast-cancer patients undergoing chemotherapy. 2011 [acesso em 2019 out 20]. Disponível em <https://www.ncbi.nlm.nih.gov/pubmed/21204889>

YASUEDA, A.; URUSHIMAET, $H$. Eficácia e interação de suplementos antioxidantes como terapia adjuvante no tratamento do câncer. 2015 [acesso em 2019 out 20]. Disponível em <https://www.ncbi.nlm.nih.gov/pubmed/19507173> 